\title{
Formal Deduction of a Volterra Series Model for Complex-valued Systems
}

\author{
C. Crespo-Cadenas ${ }^{\mathrm{a}, *}$, María J. Madero-Ayora ${ }^{\mathrm{a}}$, Javier Reina-Tosina ${ }^{\mathrm{a}}$, J. A. \\ Becerra-González ${ }^{a}$ \\ ${ }^{a}$ Departamento de Teoría de la Señal y Comunicaciones, Escuela Técnica Superior de \\ Ingeniería, Universidad de Sevilla, Spain.Tel: +34954487336.E-mail: ccrespo@us.es, \\ mjmadero@us.es, jreina@us.es, jabecerra@us.es.
}

\begin{abstract}
This paper demonstrates a general model for nonlinear systems with complexvalued inputs and its application to communication systems modeling. Based on Wirtinger calculus and a double Volterra series approach, the proposed representation can also be considered as a generalization of the widely linear transformation to incorporate the description of nonlinear systems. The complete structure is pruned with the assistance of a compressive-sensing algorithm in order to reduce the number of parameters. To illustrate this approach, it has been experimentally implemented to model a transmitter for OFDM signals, which includes an I/Q modulator and a power amplifier.
\end{abstract}

Keywords: Complex-valued Volterra models, Wirtinger calculus, double Volterra series, I/Q modulators, power amplifiers.

\section{Introduction}

Volterra series (VS) is one of the most popular representations to model modern wireless communication systems [1]. The baseband signal is used in the

\footnotetext{
${ }^{*}$ Corresponding author.

Email addresses: ccrespo@us.es (C. Crespo-Cadenas), mjmadero@us.es (María J. Madero-Ayora), jreina@us.es (Javier Reina-Tosina), jabecerra@us.es (J. A. Becerra-González)
}

Preprint submitted to Signal Processing 
modulator to generate the RF signal, possibly with linear and nonlinear impairments, and the power amplifier (PA) delivers the signal at the desired level, adding further nonlinear distortion. Without nonlinear effects, the complex envelope of the RF output signal can be viewed as a linear transformation of the complex-valued baseband signal $x[k]=x_{I}[k]+j x_{Q}[k]$. However, to exploit the complete statistical characterization of data in complex-valued signal processing, access to the information contained in the complementary correlation is required through the introduction of widely linear (WL) transformations [2][4]. When nonlinear effects need to be modeled for real-valued systems, the VS approach is a natural choice, however, its extension to the complex-valued case is not a direct mapping, and particular models are usually deduced to match specific problems. Examples of these are the proposals in array processing and beamforming [5]-[6], or in the case of impairments compensation in I/Q modulators [7], but the use of these particular models is not justified to describe the output of other systems. The availability of a general complex-valued Volterra nonlinear model is a relevant topic for the design of nonlinear communication systems. In this paper, we extend the VS approach to a general nonlinear system with complex-valued signals by using Wirtinger calculus.

\section{Volterra Models for Real-valued Systems}

For a nonlinear system described by a Volterra model with a real-valued signal $x[k]$ applied at the input, the output $y[k]$ can be written as $[8]$

$$
y[k]=h_{0}+\sum_{n=1}^{\infty} \sum_{\mathbf{q}_{n}=\mathbf{0}}^{\mathbf{Q}_{n}} h_{n}\left[\mathbf{q}_{n}\right] \prod_{r=1}^{n} x\left[k-q_{r}\right]
$$

where $h_{n}\left[\mathbf{q}_{n}\right]$ is the $n$ th-order Volterra kernel, $\prod_{r=1}^{n} x\left[k-q_{r}\right]=x\left[k-q_{1}\right] x[k-$ $\left.q_{2}\right] \cdots x\left[k-q_{n}\right], \mathbf{q}_{n}=\left[q_{1}, q_{2}, \cdots, q_{n}\right]^{T}$ is a vector of delays of the $n$ th-order term, with $q_{r}=0,1, \cdots, Q_{n}$ for all $r$, and $\mathbf{Q}_{n}=\left[Q_{n}, Q_{n}, \cdots, Q_{n}\right]^{T}$ is the vector of maximum delays.

For bivariate nonlinear systems, the output can be expressed as a double Volterra series, equation (1) of [9]. If the two input signals are $x[k]$ and $z[k]$, 
the discrete-time output is expressed as

$$
\begin{aligned}
y[k]=h_{0,0} & +\sum_{n=1}^{\infty} \sum_{\mathbf{q}_{n}=\mathbf{0}}^{\mathbf{Q}_{n, 0}} h_{n, 0}\left[\mathbf{q}_{n}\right] \prod_{r=1}^{n} x\left[k-q_{r}\right]+\sum_{m=1}^{\infty} \sum_{\mathbf{q}_{m}=\mathbf{0}}^{\mathbf{Q}_{0, m}} h_{0, m}\left[\mathbf{q}_{m}\right] \prod_{s=1}^{m} z\left[k-q_{s}\right]+ \\
& +\sum_{n=1}^{\infty} \sum_{m=1}^{\infty} \sum_{\mathbf{q}_{n}=\mathbf{0}}^{\mathbf{Q}_{n, m}} \sum_{\mathbf{p}_{m}=\mathbf{0}}^{\mathbf{P}_{n, m}} h_{n, m}\left[\mathbf{q}_{n}, \mathbf{p}_{m}\right] \prod_{r=1}^{n} x\left[k-q_{r}\right] \prod_{s=1}^{m} z\left[k-p_{s}\right]
\end{aligned}
$$

The vector of delays $\mathbf{p}_{m}$ and the vector of maximum delays $\mathbf{P}_{m}$ have been defined as in (1). The multidimensional functions $h_{n, 0}\left[\mathbf{q}_{n}\right]$ and $h_{0, m}\left[\mathbf{q}_{m}\right]$ are standard Volterra kernels of order $n$ and $m$, respectively, and the constant $h_{0,0}$ is the zeroth-order kernel. The third group of sums contains the bivariate Volterra kernels $h_{n, m}\left[\mathbf{q}_{n}, \mathbf{p}_{m}\right]$ and cross products of $x\left[k-q_{r}\right]$ by $z\left[k-p_{s}\right]$.

\section{Specific Volterra Models for Complex-valued Systems}

In wireless communication systems it is necessary a nonlinear baseband model to express the relationship between the input and the output complex envelopes. Let us review three different situations.

- Baseband PA model. The baseband model of a power amplifier can be derived from the Volterra series RF model (1) [1, 10]. In discrete-time, the baseband Volterra input-output relationship is

$$
y[k]=\sum_{m=0}^{\infty} \sum_{\mathbf{q}_{2 m+1}=\mathbf{0}}^{\mathbf{Q}_{2 m+1}} h_{2 m+1}\left[\mathbf{q}_{2 m+1}\right] \prod_{r=1}^{m+1} x\left[k-q_{r}\right] \prod_{r=m+2}^{2 m+1} x^{*}\left[k-q_{r}\right],
$$

where $n=2 m+1$ is the (odd) nonlinear order. Unlike the kernels of the real-valued Volterra case, in this model $h_{n}\left[\mathbf{q}_{n}\right]$ is symmetric under any permutation of its first $m+1$ indices, and it is also separately symmetric under any permutation of its last $m$ indices. In the literature, the model (3) is referred to as the full-Volterra (FV) model to distinguish it from other pruned baseband Volterra representations, e.g., the memoryless model. The FV model has been deduced exclusively for PAs, and direct application to other systems is not proven. 
- Beamforming. For the narrowband array processing problem, a proposal was introduced in [5] and [6]. It is advanced by establishing the vector of complex amplitudes of the signals at the output of the sensors, $\mathbf{x}_{k}$. The input-output relation is defined as

$$
y[k]=\sum_{m=1}^{M} \sum_{q=0}^{m} \mathbf{w}_{m, q}^{H}\left(\mathbf{x}_{k}^{\otimes q} \otimes \mathbf{x}_{k}^{* \otimes(m-q)}\right),
$$

where $\mathbf{w}_{m, q}$ is a complex filter and $\otimes$ denotes the Kronecker product. This approach was applied to a third-order Volterra minimum variance distortionless response beamformer, considering only polynomial terms of odd order.

- I/Q modulators. A proposal to model linear and nonlinear impairments in I/Q modulators was presented in [7]. The output $u[k]$ is given by

$$
\begin{aligned}
u[k]= & u_{0}+\sum_{n=1}^{\infty}\left\{\sum_{\mathbf{q}_{n}=\mathbf{0}}^{\mathbf{Q}_{n}} h_{n, 0}\left[\mathbf{q}_{n}\right] \prod_{r=1}^{n} x\left[k-q_{r}\right]+\sum_{\mathbf{q}_{n}=\mathbf{0}}^{\mathbf{Q}_{n}} h_{0, n}\left[\mathbf{q}_{n}\right] \prod_{r^{\prime}=1}^{n} x^{*}\left[k-q_{r^{\prime}}\right]+\right. \\
& \left.+\sum_{\mu=1}^{n-1} \sum_{\mathbf{q}_{n}=\mathbf{0}}^{\mathbf{Q}_{n}} h_{n-\mu, \mu}\left[\mathbf{q}_{n}\right] \prod_{r=1}^{n-\mu} x\left[k-q_{r}\right] \prod_{r^{\prime}=n-\mu+1}^{n} x^{*}\left[k-q_{r^{\prime}}\right]\right\} . \quad(5)
\end{aligned}
$$

This widely nonlinear (WNL) representation can be also viewed as a nonlinear extension of the WL transformation and presents analogies with the WNL Volterra beamformer (4).

Although the results reported above have shown a good performance in their respective fields, a particular method cannot be assumed to be valid in another unknown situation. The availability of a general nonlinear model that admits complex-valued input signals is mandatory in the joint modeling of the modulator and the PA in a communications transmitter, for instance.

\section{A General Volterra Model for Complex Systems}

The results concerning the output of nonlinear functions with complex arguments are not new, although they have gone largely unnoticed by the engineering 
community [4]. In particular, Wirtinger calculus (also denoted as $\mathbb{C R}$-calculus) presents an elegant approach, which allows keeping all computations and derivations in the complex domain with expressions very similar to the real-valued case, and in a more efficient time-consuming manner [3]. In a context where the input of the system $x$ is complex-valued, the corresponding output $y$ depends on $x$ and also on the complex conjugate $x^{*}$. In that case, Wirtinger calculus allows treating $x$ and $x^{*}$ as independent variables (Theorem 1 of [3]), and therefore, the double Volterra series representation can be applied. If we replace $z$ by $x^{*}$ in (2), the complex-valued output of the system is obtained as

$$
\begin{aligned}
y[k]=h_{0,0} & +\sum_{n=1}^{\infty} \sum_{\mathbf{q}_{n}=\mathbf{0}}^{\mathbf{Q}_{n, 0}} h_{n, 0}\left[\mathbf{q}_{n}\right] \prod_{r=1}^{n} x\left[k-q_{r}\right]+\sum_{m=1}^{\infty} \sum_{\mathbf{q}_{m}=\mathbf{0}}^{\mathbf{Q}_{0, m}} h_{0, m}\left[\mathbf{q}_{m}\right] \prod_{s=1}^{m} x^{*}\left[k-q_{s}\right]+ \\
& +\sum_{n=1}^{\infty} \sum_{m=1}^{\infty} \sum_{\mathbf{q}_{n}=\mathbf{0}}^{\mathbf{Q}_{n, m}} \sum_{\mathbf{p}_{m}=\mathbf{0}}^{\mathbf{P}_{n, m}} h_{n, m}\left[\mathbf{q}_{n}, \mathbf{p}_{m}\right] \prod_{r=1}^{n} x\left[k-q_{r}\right] \prod_{s=1}^{m} x^{*}\left[k-p_{s}\right] . \quad \text { (6) }
\end{aligned}
$$

Remarking that the order of the cross-terms is given by $\nu=n+m$, the corresponding summation can be calculated by adding all the terms of the same order $\nu$, with $m=1,2, \cdots, \nu-1$, and then add all orders. If we change the notation in the three sums to designate the order of the terms with the same index $n$, the relation (6) can be written as

$$
\begin{aligned}
& y[k]= h_{0,0}+\sum_{q_{1}=0}^{Q_{1,0}} h_{1,0}\left[q_{1}\right] x\left[k-q_{1}\right]+\sum_{q_{1}=0}^{Q_{0,1}} h_{0,1}\left[q_{1}\right] x^{*}\left[k-q_{1}\right]+ \\
&+\sum_{n=2}^{\infty}\left\{\sum_{\mathbf{q}_{n}=\mathbf{0}}^{\mathbf{Q}_{n, 0}} h_{n, 0}\left[\mathbf{q}_{n}\right] \prod_{r=1}^{n} x\left[k-q_{r}\right]+\sum_{\mathbf{q}_{n}=\mathbf{0}}^{\mathbf{Q}_{0, n}} h_{0, n}\left[\mathbf{q}_{n}\right] \prod_{s=1}^{n} x^{*}\left[k-q_{s}\right]+\right. \\
&\left.+\sum_{m=1}^{n-1} \sum_{\mathbf{q}_{n}=\mathbf{0}}^{\mathbf{Q}_{n-m, m}} \sum_{\mathbf{p}_{m}=\mathbf{0}}^{\mathbf{P}_{n-m, m}} h_{n-m, m}\left[\mathbf{q}_{n-m}, \mathbf{p}_{m}\right] \prod_{r=1}^{n-m} x\left[k-q_{r}\right] \prod_{s=1}^{m} x^{*}\left[k-p_{s}\right]\right\} .
\end{aligned}
$$

The complex Volterra series (CVS) model (7) is the counterpart of the realvalued Volterra series (1) when the input is complex-valued. Note that if the nonlinearity of the system can be neglected, the CVS model is reduced to the WL transformation.

Equation (7) can be written in a more compact form. To that aim, let us rearrange the elements of the $n$ th-order tensor $h_{n-m, m}\left[\mathbf{q}_{n-m}, \mathbf{p}_{m}\right]$ to form the 
vector $\mathbf{h}_{n-m, m}$ as

$$
\begin{aligned}
& \mathbf{h}_{n-m, m}=\left[h_{n-m, m}[0,0, \cdots, 0], h_{n-m, m}[0,0, \cdots, 1], \cdots,\right. \\
& \left.h_{n-m, m}\left[0,0, \cdots, P_{n-m, m}\right], \cdots, h_{n-m, m}\left[Q_{n-m, m}, Q_{n-m, m}, \cdots, P_{n-m, m}\right]\right]^{T} .
\end{aligned}
$$

Recalling that the first $n-m$ indices have maximum delays $Q_{n-m, m}$ and the other $m$ indices have maximum delays $P_{n-m, m}$ and using the definition $\mathbf{x}_{Q_{n-m, m}, k}=\left[x[k], x[k-1], \cdots, x\left[k-Q_{n-m, m}\right]\right]^{T},(7)$ can be rewritten as

$$
y[k]=h_{0,0}+\sum_{n=1}^{\infty} \sum_{m=0}^{n} \mathbf{h}_{n-m, m}^{T} \mathbf{x}_{Q_{n-m, m}, k}^{\otimes(n-m)} \otimes \mathbf{x}_{P_{n-m, m}, k}^{* \otimes m} .
$$

The CVS model is valid to describe the three examples exposed above. In the PA case, only input products with $x$ and $x^{*}$ appearing $m+1$ and $m$ times, respectively, must be considered and (7) is reduced to the baseband FV model. It is important to note that the CVS model has been demonstrated without any assumption on the internal structure of these systems and nonetheless it presents all the terms required for the nonlinear detection and estimation problem in narrowband array processing [5]-[6]. If we consider an array of $N_{s}$ narrowband sensors in (7), then $Q_{n-m, m}=P_{n-m, m}=N_{s}-1$, for all $n$ and $0 \leq m \leq n$. In that case, the CVS model (9) reduces to the WNL Volterra beamformer (4) for odd-order terms. Finally, in the case of I/Q modulators the WNL representation (5) is a particular case of the CVS model. In this paper we also refer to (7) as WNL model.

Considering the increased model complexity because of its structure with a larger number of parameters, the technique presented in [11] was used to cope with this great number of coefficients, relying on the sparsity assumption for the kernels of the model. Recalling that the WNL model is linear with respect to the coefficients, (9) can be rewritten as

$$
\mathbf{y}=\mathbf{X} \cdot \mathbf{h}+\mathbf{e}
$$

where $\mathbf{y}$ is a column vector with the samples of the output complex-envelope, $\mathbf{e}$ is a noise vector, $\mathbf{h}$ is a vector which arranges sequentially the normalized Volterra 
coefficients of the model, and $\mathbf{X}$ is a measurement matrix whose columns stack the samples of the model regressors for the same order and delay. The solution to (10) is the least squares (LS) estimate for the kernel vector

$$
\hat{\mathbf{h}}=\left(\mathbf{X}^{H} \cdot \mathbf{X}\right)^{-1} \mathbf{X}^{H} \cdot \mathbf{y},
$$

where $H$ is the Hermitian transpose operator. If only a few of the regressor coefficients are active, it is possible to apply the Orthogonal Matching Pursuit technique and the Bayesian Information Criterion aimed at determining the active support set of model coefficients [11]. This approach applied to the model provides a reduced-complexity structure which will be referred to as WNL compressed-sensing (WNL-CS) model. The particular model coefficients are still solved by using (11), but here it only applies to the subset of active regressors defining the WNL-CS structure.

\section{Application to Transmitter Modeling}

An experimental study based on a vector signal generator (VSG) and a vector signal analyzer (VSA) has been conducted. The VSG served as an I/Q modulator, providing a flexible interface to introduce I/Q impairments, and its output was fed into a commercial PA constructed with the CGH40010 GaN HEMT transistor, from Cree Inc. The test signal was a $15-\mathrm{MHz}$ orthogonal frequency-division multiplexing (OFDM) signal generated at $3.6 \mathrm{GHz}$ according to the long-term evolution (LTE) downlink standard. In the receiver side, the RF signal was measured at the VSA with a sampling rate of $92.16 \mathrm{MS} / \mathrm{s}$ providing over 360000 samples. The test signal was composed of 56 OFDM symbols and model identification was performed with only one symbol.

Following the usual methodology for behavioral modeling of transmitter architectures, model parameters were identified by applying a conventional LS procedure to the input-output measurement datasets, providing the normalized mean square error (NMSE) between the modeled and measured signal as a quality metric, in addition to the adjacent channel error power ratio (ACEPR), the latter evaluated for both the upper and lower adjacent channels. 
Table 1: Modeling performance in terms of the NMSE and ACEPR for the test PA with impairments in the I/Q modulator

\begin{tabular}{|c|c|c|c|c|c|c|c|}
\hline \multirow{2}{*}{ Model } & \multicolumn{5}{|c|}{$\operatorname{ACEPR}(\mathrm{dBc})$} & \multirow{2}{*}{$\begin{array}{c}\text { NMSE } \\
\text { (dB) }\end{array}$} & \multirow{2}{*}{$\begin{array}{c}\# \\
\text { Coeff }\end{array}$} \\
\hline & $-30 \mathrm{MHz}$ & $-15 \mathrm{MHz}$ & In-Band & $+15 \mathrm{MHz}$ & $+30 \mathrm{MHz}$ & & \\
\hline $\mathrm{FV}-\mathrm{CS}$ & -62.8 & -54.7 & -31.7 & -54.6 & -66.4 & -31.6 & 16 \\
\hline WNL-CS & -68.8 & -65.2 & -54.5 & -65.1 & -68.2 & -52.7 & 129 \\
\hline
\end{tabular}

Modeling performance of the proposed WNL approach was compared to the $\mathrm{FV}$, given its demonstrated accuracy in the context of PAs. Considering the PA operation point, both models were configured with thirteenth-order, a memory length $Q=3$ for orders one to five, and memoryless kernels for higher orders. Due to the relatively large number of coefficients in the general proposal (248 for the FV model and 1370 for the WNL model), the compressed-sensing algorithm [11] was applied to select the appropriate kernels of both models.

According to Table 1, under I/Q modulator impairments, the FV compressedsensing (FV-CS) model reduces the number of coefficients to 16 and degrades the NMSE to -31.6 dB, while the pruned WNL-CS model consists of 129 coefficients and presents an NMSE of $-52.7 \mathrm{~dB}$ that outperforms the former in about $20 \mathrm{~dB}$. While the number of the WNL-CS coefficients can be high, an increase of the FV-CS coefficients leads to overfitting because the appropriate regressors are missing. This behavior is confirmed by the predicted spectra plotted in Fig. 1, showing that the model tightly matches the measured signal, while the FV-CS model fails in the description of spectral regrowth in the adjacent channels. These outcomes are even more evident in Fig. 2, where the spectra of the error are depicted. As a reference we include in this figure the results for the test PA without I/Q modulator impairments (dash-dot line), showing a very low spectrum error inside the signal band for the FV-CS model. Let us clarify that impairments were removed from the $\mathrm{I} / \mathrm{Q}$ modulator output by using a precompensator based on [7], and therefore this case resembles a non- 


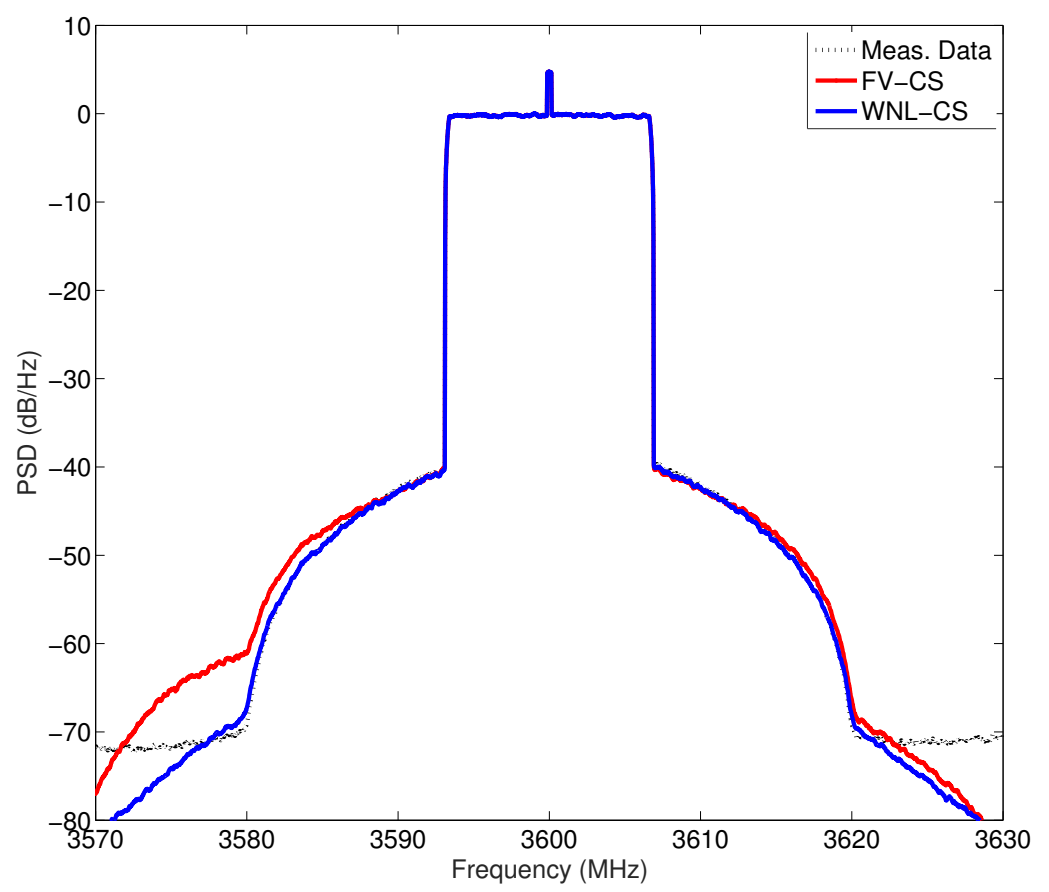

Figure 1: Normalized power spectral density of the measured (dotted line) and modeled (solid lines) output of the PA driven at 3.6 GHz with an impaired I/Q modulator.

linear system for which the baseband PA model holds. However, when I/Q impairments are present, the FV model cannot describe accurately the output and the in-band error degrades by almost $30 \mathrm{~dB}$. On the contrary, the WNL preserves the performance in that case. The spectrum of the error predicted by the WNL-CS under compensated I/Q impairments, not shown, is almost the same as the FV-CS model.

\section{Conclusions}

A formal deduction of a Volterra model for complex-valued nonlinear systems has been submitted in this paper. It is also demonstrated that the input-output relationships derived $a d-h o c$ for several communication systems -a power ampli- 


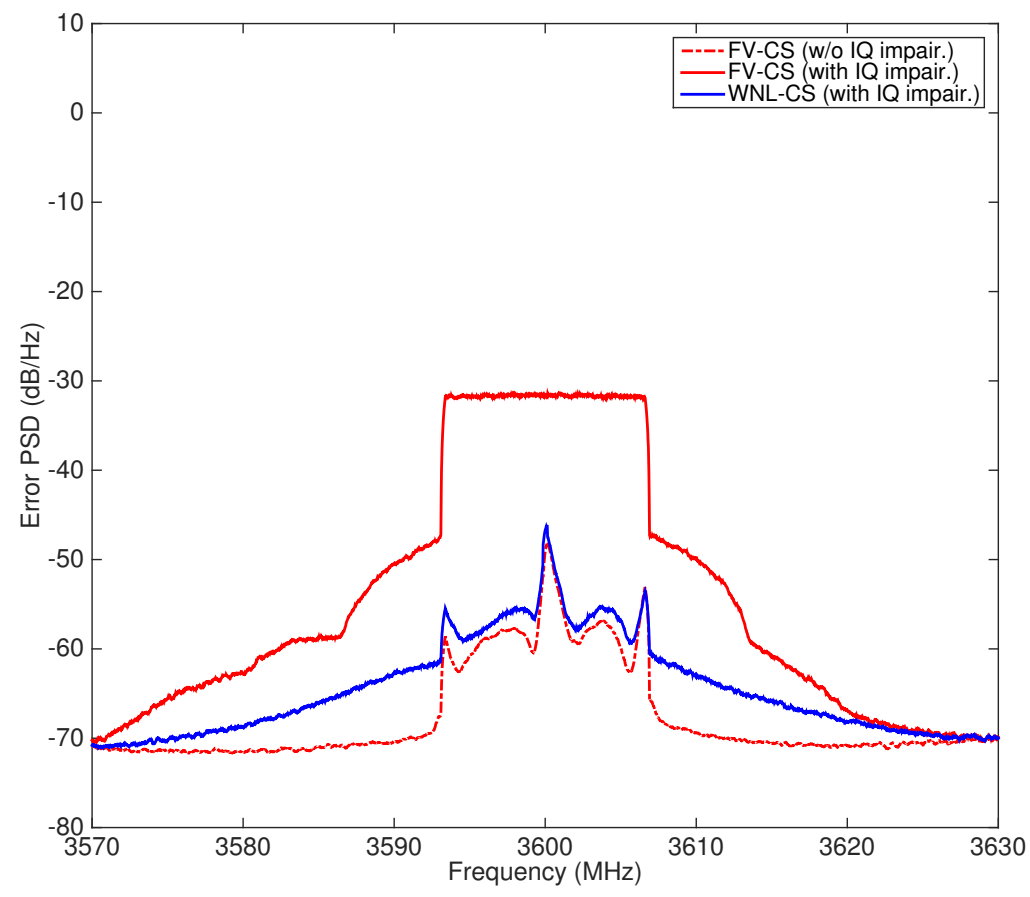

Figure 2: Spectrum of the error signal between the measured and modeled output of the PA driven at $3.6 \mathrm{GHz}$ with an impaired I/Q modulator (solid lines) and an I/Q modulator with precompensated impairments (dot-dashed line).

fier, a beamformer, an I/Q modulator- are particular cases of the CVS model. To handle the increment of the parameters, we have exploited the kernel sparsity in this approach and reduced the structure complexity with the proposed WNL-CS model. Through experimental validation, it has been also shown that this model is able to accurately represent a diverse range of distortion sources in communication subsystems, including impairments at the I/Q modulator combined with the nonlinear distortion associated to PAs. Although conventional behavioral models are accurate for the description of nonlinearities in PAs, they show a degraded performance when the input signal is impaired by a quadrature modulator. On the contrary, the richness of its regressors makes the proposed 
model a robust representation of a general transmitter-receiver architecture.

\section{Acknowledgments}

This work was supported in part by the Spanish National Board of Scientific and Technological Research (CICYT) under Grant TEC2014-53103-P.

\section{References}

[1] S. Benedetto, E. Biglieri, and R. Daffara, "Modeling and performance evaluation of nonlinear satellite links - A Volterra series approach," IEEE Trans. Aerosp. Electron. Syst., vol. 15, no. 4, pp. 494-507, Jul. 1979.

[2] B. Picinbono and P. Chevalier, "Widely linear estimation with complex data," IEEE Trans. Signal Process., vol. 43, no. 8, pp. 2030-2033, Aug. 1995.

[3] H. Li and T. Adali, "Complex-valued adaptive signal processing using nonlinear functions," EURASIP Journal on Advances in Signal Process., vol. 8, no. 2, pp. 1-9, 2008.

[4] D. Adali, P. J. Schreier, and L. L. Scharf, "Complex-valued signal processing: the proper way to deal with impropriety," IEEE Trans. Signal Process., vol. 59, no. 11, pp. 5101-5108, Nov. 2011.

[5] P. Chevalier, P. Duvaut, and B. Picinbono, "Complex transversal Volterra filters optimal for detection and estimation," in Proc. Acoust. Speech and Signal Process. (ICASSP), Toronto, ON, 1991, pp. 3537-3540.

[6] P. Chevalier, A. Oukaci, and J. P. Delmas, "Third order widely non linear Volterra MVDR beamforming," in Proc. Acoust. Speech and Signal Process. (ICASSP), Prague, 2011, pp. 2648-2651. 
[7] C. Crespo-Cadenas, M. J. Madero-Ayora, J. Reina-Tosina, and J. A. Becerra-González, "A widely nonlinear approach to compensate impairments in I/Q modulators," in Proc. 2015 Eur. Microw. Conf., Paris, France, 6-11 Sep. 2015, pp. 506-509.

[8] V. J. Mathews and G. J. Sicuranza, Polynomial Signal Processing. New York: Wiley, 2000.

[9] S. O. Rice, "Volterra systems with more than one input port - Distortion in a frequency converter," Bell Syst. Techn. J., vol. 52, no. 8, pp. 1255-1270, Oct. 1973.

[10] R. Raich and G. T. Zhou, "On the modeling of memory nonlinear effects of power amplifiers for communication applications," in Proc. 10th Dig. Signal Proc. Workshop, 2002, pp. 7-10.

[11] J. Reina-Tosina, M. Allegue-Martinez, C. Crespo-Cadenas, Y. Chao, and S. Cruces, "Behavioral modeling and predistortion of power amplifiers under sparsity hypothesis," IEEE Trans. Microw. Theory Techn., vol. 63, no. 2, pp. 745-753, Feb. 2015. 